\title{
Simulating the human motion under Functional Electrical Stimulation using the HuMAnS toolbox
}

\author{
M. Eckert ${ }^{(1)}$, M. Hayashibe ${ }^{(2)}$, D. Guiraud ${ }^{(2)}$, P-B Wieber ${ }^{(3)}$, P. Fraisse ${ }^{(2)}$ \\ (1)IMERIR, Avenue Paul Pascot, BP 90443, 66004 Perpignan Cedex, France, \\ Phone: +33 468568018 Fax: +33 468550386 Email: martine.eckert@imerir.com \\ ${ }^{(2)}$ DEMAR Project, INRIA - LIRMM, 161 rue Ada, 34392 Montpellier Cedex 5, \\ France \\ Phone: +33 $467418621 \quad$ Fax: +33 467418500 \\ Email: mitsuhiro.hayashibe@lirmm.fr, david.guiraud@lirmm.fr, fraisse@lirmm.fr \\ ${ }^{(3)}$ BIPOP Project, INRIA Grenoble - Rhône-Alpes, Inovallée, 655 avenue de \\ l'Europe, Montbonnot, 38334 Saint Ismier Cedex France
}

Phone: +33476615392Ｆax:+33476615477Ｅmail: pierre-brice.wieber@inrialpes.fr

\begin{abstract}
:
Mathematical models of the skeletal muscle can support the development of neuroprotheses to restore functional movements in individuals with motor deficiencies by the mean of Functional Electrical Stimulation (FES). Since many years, numerous skeletal muscle models have been proposed to express the relationship between muscle activation and generated force. One of them (Makssoud et al [2]-[3]), integrates the Hill model [5] and the physiological one based on Huxley work [4] allowing the muscle activation under FES. We propose in this paper an improvement of this model by modifying the activation part. These improvements are highlighted through the HuMAnS toolbox [1] using a 3D biomechanical model of human named Human 36. This article describes this toolbox and the software implementation of the model. Then, we present the results of the simulation.
\end{abstract}

Keywords: Simulation, 3D biomechanical model of Human, models of skeletal muscles, Functional Electrical Stimulation.

\section{Abbreviations: \\ FES: Functional Electrical Stimulation \\ HuMAnS toolbox: Humanoid Motion Analysis and Simulation toolbox [1] \\ Introduction}

Different approaches to model the electromechanical behavior of skeletal muscles with FES signal as inputs have been presented in previous studies [2]-[3]. Thanks to the development of biomechanics, different musculoskeletal human models have also been developed and available. The general musculoskeletal model of whole body and its dynamics computation method were established [7]-[8]. The effective computational algorithms were pursued for inverse and forward dynamics on the basis of efficient multibody dynamics-computation algorithms 
[6]. In these works, Hill-type model [9] is normally used for the calculation of muscle force. In the HuMAnS toolbox (Humanoid Motion Analysis and Simulation toolbox developed at the INRIA Rhône-Alpes [1]), a 3D biomechanical model of the human has been developed: the Human 36 model in which the mathematical muscle model proposed by DEMAR project [2] has been introduced. In this muscle model, Hill representation and physiological model based on the work of Huxley [4] are integrated and designed especially for muscle activation through FES.

Using the HuMAnS toolbox, we have modified the initial model in order to improve the output mechanical response. Input command and the force-length relationship were thus enhanced. Then, we observe the results of the modifications. In the first part of this article, we give a description of the HuMAnS toolbox and particularly the Human 36 model. Then, we describe the two models of the skeletal muscle we use, and we present the results and discussion of the simulation.

\section{Materials and Methods}

\section{The HuMAnS toolbox and the Human 36 model}

The HuMAnS toolbox developed at INRIA Rhône-Alpes offers tools for the modelling, the control and the analysis of humanoid motion, being that of a robot or a human. It is an Open Source software. It is composed of two parts: a first one which is the model generation and a second one which is the simulation, the control and the analysis tools. A 3D biomechanical model of human has been developed on this software: the Human 36 model.

The Human 36 model includes the geometric, the kinematic and the dynamic model of the whole human body and also the interaction of the body with the environment [10]. The dynamic model is a lagrangian model [1]. The interest of using the Human 36 model for static or dynamics studies has been proved in a previous work [11].

The mathematical model of the skeletal muscle proposed by DEMAR project for FES applications has also been implemented in the Human 36 model. Initially, it is the model proposed by El Makssoud et al [2]-[3]. As HuMAnS is an Open 
Source software, we are able to change the skeletal muscle model and to observe the results of the modifications.

\section{Skeletal muscle models}

The muscle models we proposed are composed of two parts of different nature [3]: first, an activation model describing how an electrical stimulus generates an Action Potential (AP) and initiates the contraction and recruits Motor Units, then a mechanical model describing the generation of forces and an evolution of lengths [3] (Figure 1).

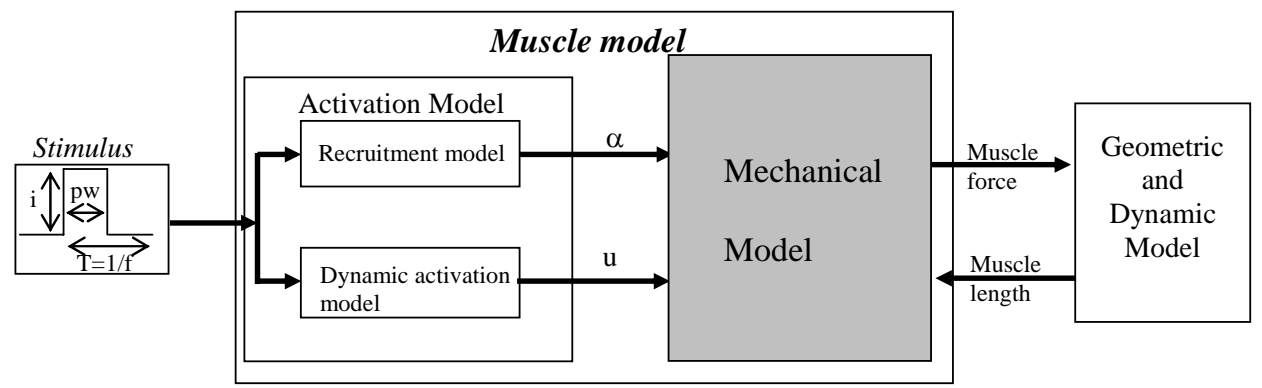

Figure 1. Electromechanical model of the muscle

The contraction force generated by a muscle under FES is mainly modulated therefore by the stimulation parameters: intensity (i) and pulse width (pw). For this reason, the muscle model has these stimulation parameters as inputs, the generated force and active stiffness as outputs.

\section{The skeletal muscle model initially implemented in Human 36}

The command signal $\mathrm{u}(\mathrm{t})$ is shown on the Figure 2. It fluctuates between two values $U_{p}$ and $U_{m}$. Its different states correspond to different activations: contraction phase (2), transition phase (1) and relaxation phase (0) (Figure 2) [2].
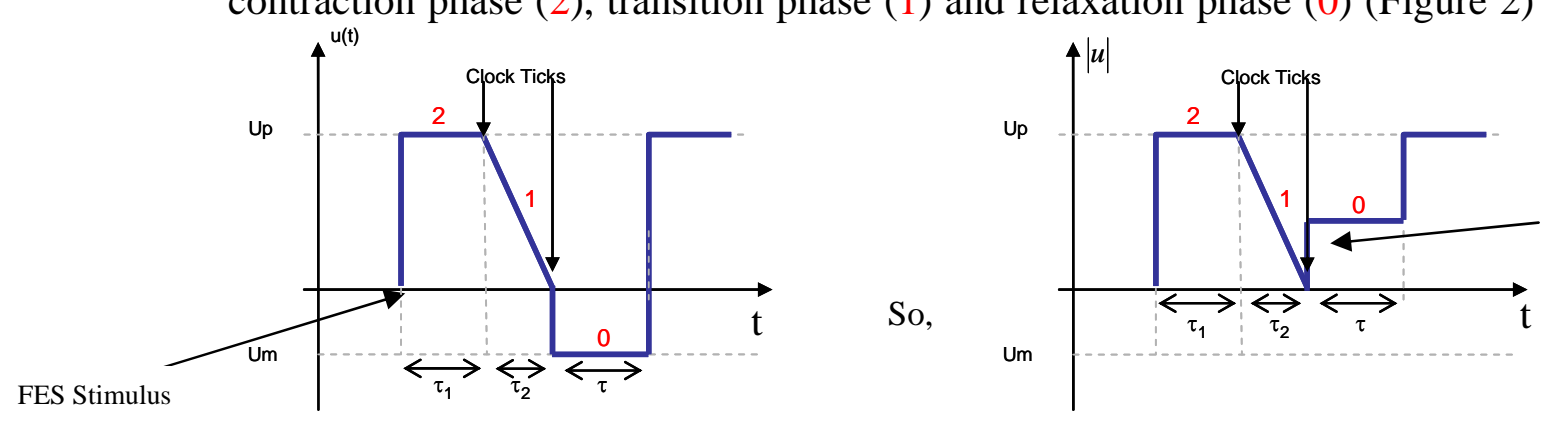

Figure 2. Command signal [1]

The recruiting rate depends on the width and intensity of the impulsion. Its value is given by [2]: 


$$
\alpha=\frac{1}{2}+\frac{1}{2} \frac{\tanh \left(5\left(\frac{p w i}{p w_{\max } i_{\max }}-\frac{1}{2}\right)\right)}{\tanh \left(\frac{5}{2}\right)}
$$

The mechanical model is composed of: a contractile component with the command signal $\mathrm{u}(\mathrm{t})$ and $\alpha$ as inputs, and passive elements composed of a serial linear spring and a parallel exponential spring (Figure 3)[2].

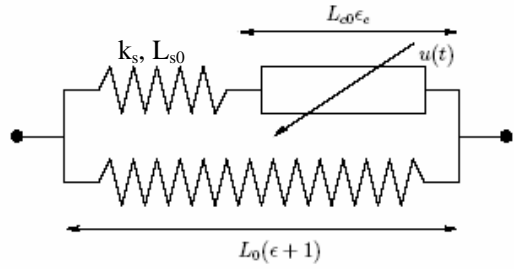

Figure 3. Mechanical Model of the skeletal muscle [1]

The stiffness and the force of a muscle depend on its length:

$$
k_{0}=k_{m} e^{-\left(\frac{\varepsilon_{c}}{0.4}\right)^{2}} \quad F_{0}=F_{m} e^{-\left(\frac{\varepsilon_{c}}{0.4}\right)^{2}}
$$

For the quadriceps and hamstrings muscles, the $\mathrm{k}_{\mathrm{m}}, \mathrm{F}_{\mathrm{m}}, \mathrm{k}_{\mathrm{s}}, \mathrm{k}_{\mathrm{p}}, \mathrm{k}_{\mathrm{ep}}, \mathrm{L}_{\mathrm{c} 0}$ and $\mathrm{L}_{0}$ values are given in the table 1 .

\begin{tabular}{|l|l|l|l|l|l|l|l|}
\hline & $\mathrm{k}_{\mathrm{m}}$ & $\mathrm{F}_{\mathrm{m}}$ & $\mathrm{k}_{\mathrm{s}}$ & $\mathrm{k}_{\mathrm{p}}$ & $\mathrm{k}_{\mathrm{ep}}$ & $\mathrm{L}_{\mathrm{c} \odot}$ & $\mathrm{L}_{\odot}$ \\
\hline Quadriceps & $10^{4} \mathrm{~N} \cdot \mathrm{m}^{-1}$ & $500 \mathrm{~N}$ & $10^{4} \mathrm{~N} \cdot \mathrm{m}^{-1}$ & $\odot$ & $1 \mathrm{~N} \cdot \mathrm{m}^{-1}$ & $8.2 \mathrm{~cm}$ & $49.2 \mathrm{~cm}$ \\
\hline Hamstrings & $10^{4} \mathrm{~N} \cdot \mathrm{m}^{-1}$ & $500 \mathrm{~N}$ & $10^{4} \mathrm{~N} \cdot \mathrm{m}^{-1}$ & $\odot$ & $1 \mathrm{~N} \cdot \mathrm{m}^{-1}$ & $10,7 \mathrm{~cm}$ & $49.2 \mathrm{~cm}$ \\
\hline
\end{tabular}

Table 1. Maximum stiffness and force of quadriceps and hamstrings muscles

The dynamic model of contractile component coupled with the linear series spring (Figure 3) is [2] (where $\mathrm{k}_{\mathrm{c}}, \mathrm{F}_{\mathrm{c}}, \varepsilon_{\mathrm{c}}$ are the state variables):

$$
\left\{\begin{array}{c}
\dot{k}_{c}=-\left(|u|+\left|\dot{\varepsilon}_{c}\right|\right) k_{c}+\alpha k_{0}|u|_{+} \\
\dot{F}_{c}=-\left(|u|+\mid \dot{\varepsilon_{c}}\right) F_{c}+\alpha F_{0}|u|_{+}+k_{c} L_{c 0} \dot{\varepsilon}_{c} \\
\dot{F}_{c}=k_{s}\left(L_{0} \dot{\varepsilon}-L_{c 0} \dot{\varepsilon}_{c}\right)
\end{array}\right.
$$

Then, we can compute $\dot{\varepsilon}_{c}$ from the command u, the recruiting rate $\alpha$, the forces $\mathrm{F}_{\mathrm{c}}$ and $F_{0}$, the stiffness $k_{c}$ thanks to the first and last equations of the system (eq. 3): $\dot{\varepsilon}_{c}=\frac{k_{s} L_{0} \dot{\varepsilon}+F_{c}|u|-\alpha F_{0}|u|_{+}}{k_{s} L_{c 0}+k_{c} L_{c 0}-S_{\varepsilon_{c}} F_{c}} \quad$ (with $S_{\varepsilon_{c}}$ the sign of $\dot{\varepsilon}_{c}$ ) (eq. 4)

The total force developed by the muscle is: $\quad F=F_{c}+\frac{k_{p}}{k_{e p}}\left(e^{k_{e p} \varepsilon}-1\right)$ 


\section{New activation model}

We propose here an evolution of the DEMAR skeletal muscle model presented above. This new model has been developed directly from the Huxley [4] and the Hill models [5] and its parameters estimated on animal muscles.

Compared to the previous ones, it includes a new recruitment function and more accurate dynamics, including a force-length relation at the microscopic scale.

A new command signal $\mathrm{u}(\mathrm{t})$ is implemented (Figure 4).

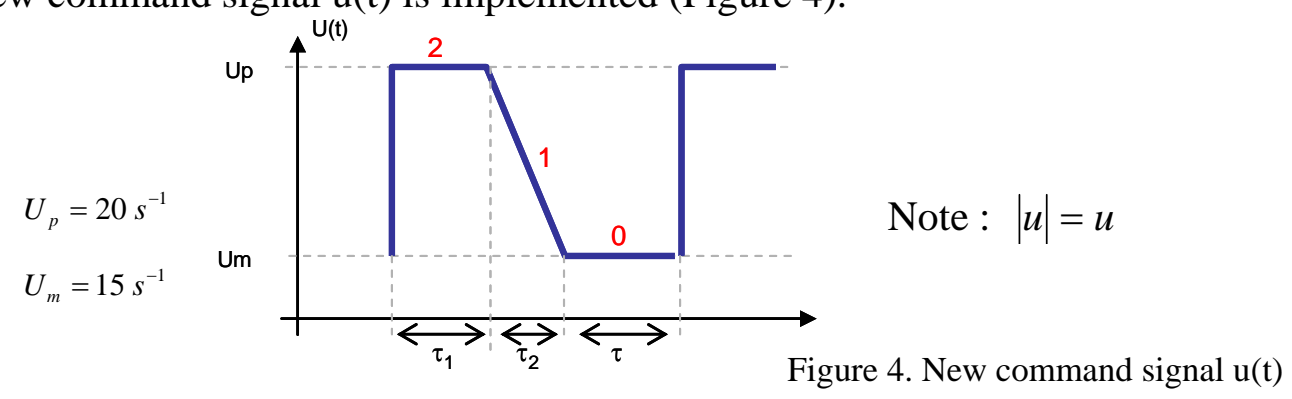

The contraction-relaxation cycle is considered therefore to stick to the previous simple three phases.

The stronger the intensity (i) and the longer of the pulse width (pw), the bigger the contraction of the muscle. We propose therefore a new recruitment function with these two parameters partially decoupled:

$$
\alpha(p \omega, i)=d(\tanh (R-c)+\tanh (c)) \quad R=b\left(1+a_{p \omega} \frac{p \omega}{p \omega_{\max }}\right)\left(1+a_{i} \frac{i}{i_{\max }}\right)\left(\frac{q}{q_{\max }}\right)
$$

\section{Implementation and Results}

The new model presented above has been implemented in the Human 36 model [1]. This part describes the results obtained.

\section{Modification of the Activation Model}

We have modified the shape of $\mathrm{u}(\mathrm{t})$ and $\alpha$ in the initial model. Only four muscles are modeled: the quadriceps and the hamstrings of the two legs.

We have applied a Swing Up regular movement to the human 36 model. That means that at the beginning, the human body is standing and the legs are straight. Then, for the two legs, we stimulate the hamstrings during 30ms to the bending position after we simulate the quadriceps during 30ms to the extension position and so on... 
The movement is calculated for both knees. For the right knee, a free movement is performed whereas for the left knee isometric case is studied.

The results concerning the rotation angle of the right knee (Q) (the left knee doesn't move), the torques, the active stiffness $\left(k_{c}\right)$, the force $\left(F_{c}\right)$ and $\varepsilon_{c}$ of the contractile element are presented on the Figure 5.

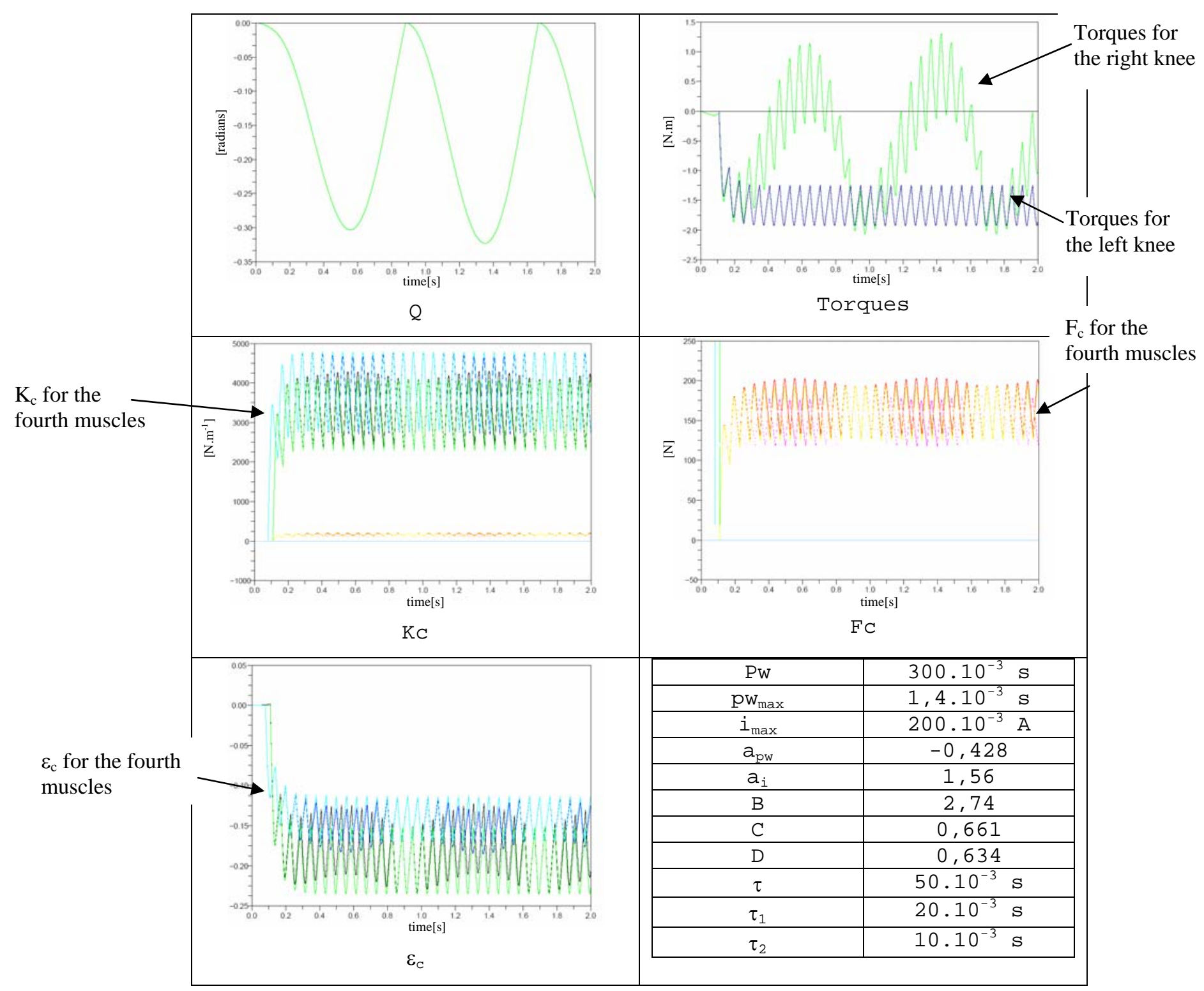

Figure 5. Evolution of the angle of rotation (Q), the torques, the contractile stiffness (Kc) and the contractile force $(\mathrm{Fc})$ when the new activation model is implemented with the parameters

The first model we proposed has been modified due to a bad behavior while performing simulation. It was due to two main problems we dealed with: the $|u|$ that was discontinued, and the recruitment curve that can not render the difference of recruitment effect depending on the input used to modulate force (i or pw). We thus change the shape of $\mathrm{u}$ without modifying the physiological meaning of this control. For the recruitment, we propose a new recruitment function that takes into 
account the difference between $\mathrm{i}$ and pw modulation. Moreover, in the new model, the recruitment $\alpha$ is kept to 0 when either i or pw are 0 and its cross sections along the $\mathrm{i}$ and pw axes follow sigmoid like functions as observed experimentally when varying only one of these parameters. Thus both modifications enhance the model with a more realistic behavior, validated on real experiments [12].

As regards the simulation results we can notice the original following results: i) the model and its implementation is able to render both isometric and non isometric contractions, Hill type models can hardly achieve this with the same set of identified parameters, ii) Relaxation and contraction phases dynamics are different as in the real world, this is also not render by linear models of the muscle, iii) internal variable such as active stiffness, relative length of the contractile element can be observed and simulated, again, classical Hill-type models can not give such pieces of information, iv) finally, as contraction and relaxation phases dynamics can be adjusted separately, and owing to the $\mathrm{u}$ command input, different from the recruitment input, the model is able to predict both twitch and titanic contraction with a high accuracy. Figure 5 shows the results obtained, consistent with experimental data (not presented here).

This analysis of the model concerns the software implementation issue, quite complex to achieve, mainly due to the switching and initialization of differential equations and the coupling between muscle's equation and biomechanics state variables such as length. We also qualitatively validate the global behavior of the HuMAnS software with such complex simulation, through the validation of known properties of muscle’s behavior listed above.

\section{Conclusion and Perspectives}

An initial skeletal muscle model for FES applications was proposed by DEMAR project and has been implemented in the Human 36 model (HuMAnS toolbox [1]). As HuMAnS gives the possibility to test and different models of muscle, we simulate here an evolution of the activation model of this model, which is more in accordance with the physiological muscle behavior.

Even though we have already validated this model through experiments on rabbit's muscles, we will be able now to introduce the other skeletal muscles of the human body in order to use the Human 36 model to compare the results given 
by the simulation and the results obtained with experiments on subjects performing induced FES movements. The model is also able to simulate voluntary contraction but it needs to be modified in order to accept EMG signals as inputs instead of FES parameters. This work is already under development.

\section{Acknowledgements}

Thanks to C.Azevedo-Coste, M.Benoussaad and R.Mozul for their precious help in this work.

\section{References:}

[1] Wieber P.B., Billet F., Boissieux L., Pissard-Gibollet R. (2006), "The HuMAnS toolbox, a homogeneous framework for motion capture, analysis and simulation”, Ninth International Symposium On The 3D Analysis Of Human Movement, June 2006.

[2] H. El Makssoud, "Modélisation et Identification des Muscles Squelettiques sous Stimulation Electrique Fonctionnelle”, Phd thesis, Université Montpellier 2, 2005.

[3] H. El Makssoud , D. Guiraud, P. Poignet "Mathematical Muscle Model for Functional Electrical Stimulation Control Strategies", Proceedings of the International Conference on Robotics and Automation (ICRA), New Orleans, USA, April 2004, p. 1282-1287.

[4] A.F.Huxley, Muscle structure and theories of contraction, "Progress in Biophysics and Biophysical Chemistry”, vol. 7, pp. 255-318, 1957.

[5] A.V.Hill, "The heat of shortening and the dynamic constants in muscle", Proceeding of the Royal Society, London, Sre. B, vol. 126, pp. 136-195, 1938.

[6] Yoshihiko Nakamura, Katsu Yamane, Yusuke Fujita, Ichiro Suzuki, "Somatosensory Computation for Man-Machine Interface From Motion-Capture Data and Musculoskeletal Human Model”, IEEE Transactions on Robotics, vol.21, No.1, February 2005, pp. 58-66

[7] S. L. Delp and J. P. Loan, "A graphics-based software system to develop and analyze models of musculoskeletal structures”, Comput. Biol. Med., vol. 25, pp 21-34, 1995.

[8] Scott L. Delp, Frank C. Anderson, Allison S. Arnold, Peter Loan, Ayman Habib, Chand T. John, Eran Guendelman, and Darryl G. Thelen, "OpenSim: Open-Source Software to Create and Analyse Dynamic Simulations of Movement”, IEEE Transactions on Biomedical Engineering, vol. 54, No. 11, November 2007, pp. 1940-1950

[9] F.E. Zajac "Muscle and tendon: properties, models, scaling and application to biomechanics and motor control", CRC Critic. Rev. in Biomed. Eng. 17: 359-411, 1989.

[10] Wieber P.B., "Modélisation et commande d'un robot marcheur anthropomorphe", Thèse, Ecole des mines de Paris, 2000, 91 pages.

[11] Bottecchia S., "Estimation de posture : vers la prise en compte de la $3^{\circ}$ dimension sur le modèle biomécanique”, Rapport de stage de Master II Recherche, Lirmm, Montpellier, 2006, 64p.

[12] M. Hayashibe, P. Poignet, D, Guiraud, H. Makssoud, « Nonlinear Identification of skeletal muscle dynamics with Sigma-Point Kalman Filter for model-based FES », Proc. IEEE/Int. Conf. on Robotics and Automation, pp 2049-2054, 2008.

\section{List of figures}

Figure 1. Electromechanical model of the muscle p3

Figure 2. Command signal [1] $\quad$ p3

Figure 3. Mechanical Model of the skeletal muscle [1] p4

Figure 4. New command signal $\mathrm{u}(\mathrm{t}) \quad \mathrm{p} 5$

Figure 5. Evolution of the angle of rotation (Q), the torques, the contractile stiffness (Kc) and the contractile force $(\mathrm{Fc})$ when the new activation model is implemented with the parameters $\mathrm{p} 6$

\section{List of tables}

Table 1. Maximum stiffness and force of quadriceps and ischio muscles 